\title{
The effectiveness of pelvic floor exercises on symptoms in females with stress urinary incontinence
}

\author{
Nitesh Malhotra ${ }^{1 *}$ and Aksh Chahal ${ }^{* *}$ \\ ${ }^{1}$ Ph.D Scholar, Department of Physiotherapy, Shri Jagdishprasad Jhabarmal Tibrewala University, Churela, \\ Rajasthan 333001 \\ ${ }^{2}$ Department of Physiotherapy, Shri Jagdishprasad Jhabarmal Tibrewala University, Churela, Rajasthan \\ 333001 India
}

\begin{abstract}
The aim of the present investigation is to depict the interventions of pelvic floor exercises in urinary incontinence. The present study deals with the employment of pelvic floor exercises in subjects of varying age group from 30-60 years. Tools employed for the study are B-SAQ validation, the Bladder Control Self-Assessment Questionnaire (B-SAQ) and $1 \mathrm{Hr}$ pad test which was working as outcome measure. The study was designed to investigate the effect of pelvic floor exercises on symptoms and botherness caused by urinary incontinence and achieving continence level was marked through pad test. Results confirmed that there was a significant improvement in the symptoms scores of experimental group in which pelvic floor muscles were employed in contrast with the control group. Pelvic floor muscles should be done under the supervision of physiotherapist as geriatric population needs some external support in order to complete the pelvic floor exercise protocol.
\end{abstract}

\section{INTRODUCTION}

Urinary incontinence is commonest problem in old age affecting social, psychological and physical daily activities. The most prevalent urinary incontinence amongst females is Stress urinary incontinence especially at the age between $30-60$ years. Generally it is believed these reduced in muscle strength of urethra and muscle around the sphincter is responsible for causing incontinence. The intension of doing pelvic floor exercises is to increase maximal urethral pressure and also increase in reflex contractions sphincteric unit which can sustain the rise in the intraabdominal pressure. Pelvic floor muscle training exercises is the recognized and preferred

\section{ARTICLE INFORMATION:}

Corresponding Authors: malhotra.nitesh@gmail.com, aksh.chahal@gmail.com

Received 12 $2^{\text {th }}$ Aug, 2018

Accepted after revision $21^{\text {st }}$ Nov, 2018

BBRC Print ISSN: 0974-6455

Online ISSN: 2321-4007 CODEN: USA BBRCBA Thomson Reuters ISI ESC / Clarivate Analytics USA

Mono of Clarivate Analytics and Crossref Indexed Journal Mono of $C R$

NAAS Journal Score 2018: 4.31 SJIF 2017: 4.196

(-) A Society of Science and Nature Publication, Bhopal India 2018. All rights reserved.

Online Contents Available at: http//www.bbrc.in/

DOI: $10.21786 / \mathrm{bbrc} / 11.4 / 19$ 
conservative treatment for urinary incontinence, (Sapsford et al. 2001, Ferreira et al. 2004, Sinclair et al. 2011, Kılıcin et al. 2016 and Aarthi et al., 2018).

Exercises for pelvic floor were introduced in 1948 by Kegel, till date many randomized controlled studies and systematic reviews have supported the efficacy of these exercises, (Cavkaytar et al. 2014). Many researchers have reported that more than $68.4 \%$ of the women suffered from SUI while $41.2 \%$ of the women reported with mixed urinary incontinence, both showed improvement after 8 weeks of Kegel's exercises. The incidence of urinary incontinence in female subjects in a Turkish community was determined and stress urinary incontinence was found to be higher i.e. $33.7 \%$ than any other type of incontinences in females of different age groups. This was explained through observational studies, based on questionnaires, where it was found that out of total of 192 subjects having incontinence, 45.5\% had stress urinary incontinence (SUI), $19.8 \%$ had urge urinary incontinence and 34.6\% had mixed incontinence (Bhanupriya et al. 2015 and Kilıcin et al. 2016).

In the previous studies as explained with different outcome measures and exercise protocols including other intervention to enhance the muscle contraction like biofeedback, duration and number of contraction per day can also be a cause for difference in results output. Therefore pelvic floor exercises are accepted as an efficient intervention for SUI, many queries related to protocol adapted are still not yet explained. Other adjunct treatment like interferential therapies did not prove beneficial over pelvic floor exercises in SUI as recently reported by Aarthi et al. 2018).

For application of a competent treatment concern should be not only towards the pathology but also towards the social, socio-economical status and emotional aspect. Earlier surgical intervention was usually considered as foremost choice of treatment, last few years inclination towards conservative treatment has increased. As per the opinion from The International Continence Society, conservative treatment should be the first choice in incontinent patients (Dumoulin et al. 2016).

Researchers also employed different type of exercises protocol for primiparous women and their sexual efficacy by employing 8 weeks of pelvic floor exercises and found improvement in the outcome measure and strength of pelvic floor muscles (Luginbuehl et al. 2015, Malhotra et al 2018). Aforementioned studies employed for Pelvic floor exercises combination with additional treatment but there is limited study on the age group 30 - 60 years of ages. Therefore, there is need to determine that whether the stress urinary incontinence can alone be treated with PFMT on different age groups. The aim of the study is to evaluate the effectiveness of pelvic floor muscle exercise in female subjects with different age groups having stress urinary incontinence

\section{MATERIAL AND METHODS}

The 42 female subjects between the ages of 30-60 were included in the study and were divided into two groups, Control (Group A) \&t Experimental (Group B) and. The study was performed at RLJT Hospital \&t Research Centre, Jhunjhunu. The group allocation was done blinded through randomly and data acquisition, data reduction, data analysis was been blinded, the demographic data of name, age, height weight and BMI were recorded subjects present with stress urinary incontinence based on patients history ,both parous and nulliparous, pre and post menopausal and hemodynamicaly stable and physically fit for therapeutic exercises these subjects who were included in study, subjects having any history of organ prolapse, suffering from vaginal or urinary tract infection, tumors or infectious disease or under some kind of medications affecting incontinence were excluded. Total study period was for 8 weeks, readings were taken at baseline, on $4^{\text {th }}$ week and $8^{\text {th }}$ week of the study. Experimental groups were asked to come are to come every weekends and perform the exercise and follow up while control group B were only explained the exercises. Also both groups were asked to follow up on $4^{\text {th }}$ and $8^{\text {th }}$ weeks.

B-SAQ validation, the Bladder Control Self-Assessment Questionnaire (B-SAQ) and $1 \mathrm{Hr}$ pad test was employed as outcome measure, following the method of Sahai et al (2014). The B-SAQ contained 8- questions that assessed the symptoms such as number of times a patient is required to void, difficulty in holding urine, nocturia and urine leaks, for which there were associated scores. Responses to both were scored on a 4-point Likert scale. Total of both the score can give a significant illustration of patients to seek help or medical advice.

1 hour pad test: the test was performed on all the female subjects, where they were given pre-weighed pads and were asked to wear the same, few subjects were requested to wear two pads due to increased symptoms of incontinence. All subjects were asked to drink $500 \mathrm{ml}$ of plain water at room temperature in $15 \mathrm{~min}$ utes of span, following which they asked to conduct certain activities like simple walking, climbing up and down a flight of stairs, standing up-down from sitting (10 times), cough vigorously (10 times), on spot jog for 1 minute, pick up objects with bending (5 times) and washing hands in running water ( 1 minute). All patients were strictly asked not to void their bladder for 1 hour and later the pads were weighed, for which the calculations were done on the basis of pad weight in grams i.e. 
1-10 gms signified mild incontinence, 11 to 50 gms signified moderate and more than 50 gms indicated severe incontinence respectively.

Patients were explained about the anatomy and physiology of pelvic floor muscle a group class was conducted for both the groups on another day was an live demonstration video to explain how to perform the exercises in the protocol, also exercises in three different position i.e. Lying, sitting and standing an muscle contraction and stopping while passing the urine For experimental group the patients were explained and asked to perform correct pelvic floor muscle exercise in three different positions: Lying down supine with one pillow below the hips, sitting on chair and standing with legs both slightly kept apart. Patients were asked to perform exercises in lying position for first two weeks 10- 15 repetition. Contracting the muscle with a hold of 2-4 seconds of hold in each contraction without holding breath they were also asked to perform 2-3 sets per, with minimum of 20-30 contractions in a day. At $3^{\text {rd }}$ and $4^{\text {th }}$ week there was an addition of sitting position, number of set remained the same, by increasing the number of repetition to 30-40 in each set and 60-120 contractions over all throughout the day. Remaining 4 weeks, the protocol was added with an additional position of standing and keeping the number of sets as constant, the contraction per set was increased to 50-60 per set and minimal number of contractions per day ranged from 150 to 180. Telephonic and message reminders were sent on phone to encourage the subjects twice a week and queries were noted down and answered either on telephone or during the follow up visits.

\section{RESULTS AND DISCUSSION}

The data analysis was done with help of SPSS software version 19 was applied, To isolate the variable that differ from the others Turkey test was applied for multiple comparison analyze the mean and standard deviation within group and ANOVAs test were applied to calculate the difference between groups. Forty subjects were included in the study having a mean age of $46.05 \pm 6.04$ and were divided in experimental and $41.15 \pm 7.12$ in control group. The mean height in respective groups were $162 \pm 6.69 \mathrm{~cm}$ and $58.55 \pm 5.09 \mathrm{~cm}$, mean weight was $64 \pm 6.50$ and $67.3 \pm 5.54 \mathrm{in} \mathrm{kg}$, The mean BMI was also calculated as $25.23 \pm 2.44$ and $24.63 \pm 2.13$ respectively for experimental and control group.

Amongst the 42 participant in the study, 2 women could not complete the study there was no significant difference seen in Group A (Control) before treatment and after treatment, but there was a statistical difference seen amongst Group B (Experimental Group) subjects at 4 th week and $8^{\text {th }}$ week of treatment.

As shown in figure I, the pad test demonstrates the significant improvement at $4^{\text {th }}$ and $8^{\text {th }}$ week for patient who was engaged in exercises and with regular followups Group B, there was an improvement seen in the pad test performed for women in control group (Group A) but was not statistically significant.

Explaining through figure $1 \mathrm{Et}$ table 2 there was no significant difference found in between baseline data and $4^{\text {th }}$ week and at end of $8^{\text {th }}$ week in Group A (Control group), similarly when compared within intergroups as explained through table 3 , there was a significant dif-

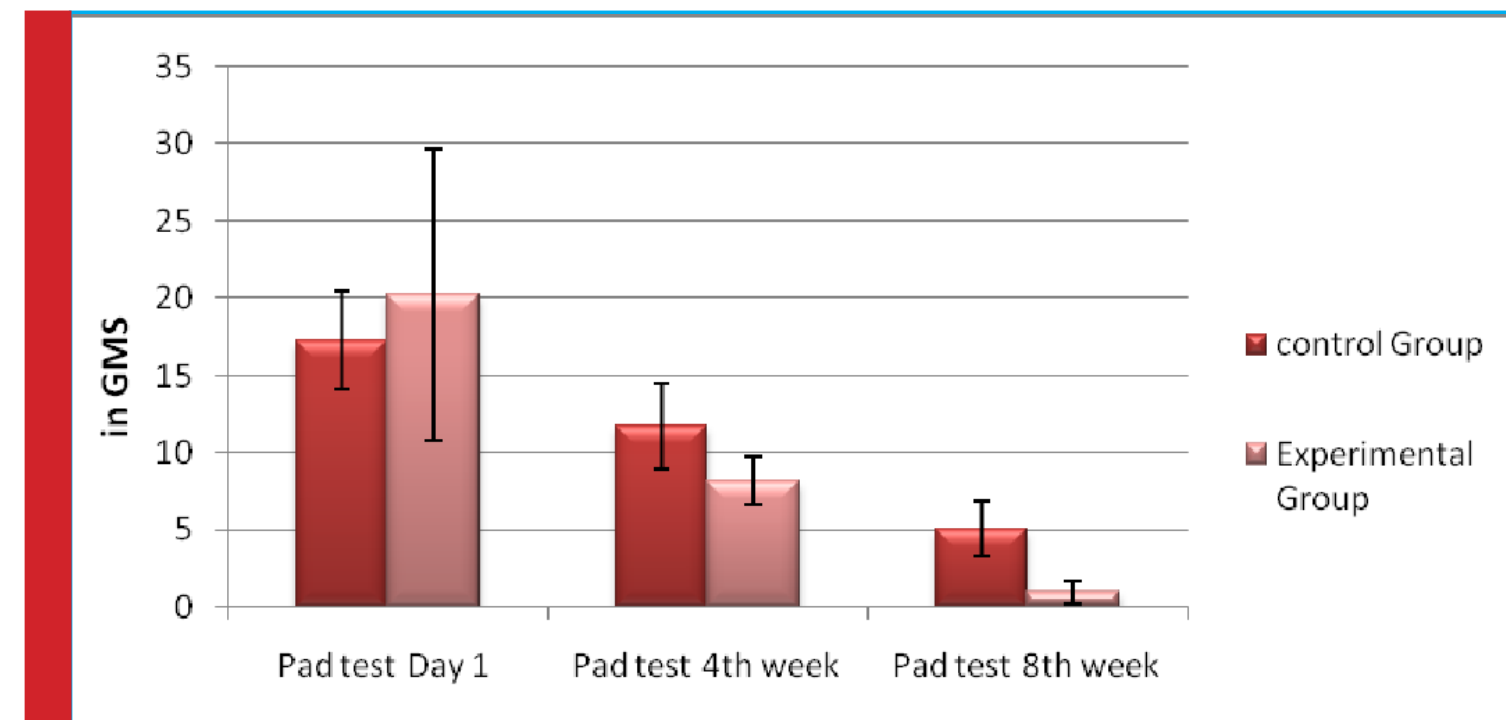

FIGURE 1. Illustrates about the mean and standard deviation difference in the score in Pad test 
Table 1. Illustrates the mean and standard deviations in demographic data.

\begin{tabular}{|l|l|l|}
\hline Characteristics & Group A & Group B \\
\hline Age (Mean \pm SD) & $41.15 \pm 7.12$ & $46.05 \pm 6.04$ \\
\hline Height (In cm) (Mean \pm SD) & $158.55 \pm 5.09$ & $162 \pm 6.69$ \\
\hline Weight(In kg) (Mean \pm SD) & $67.3 \pm 5.54$ & $64 \pm 6.50$ \\
\hline BMI (Mean \pm SD) & $24.63 \pm 2.13$ & $25.23 \pm 2.44$ \\
\hline
\end{tabular}

ference seen in pad test results for experimental groups, bringing the $\mathrm{P}$ at significant value of $<0.001$.

Explaining through figure 2 the result signifies that symptoms score of B-SAQ were significantly improved from day $14^{\text {th }}$ week and at $8^{\text {th }}$ for group B week but there no significant improvement in the scores for control group, figure 3 explains about the bothers score in B-SAQ in shows neither the experimental group nor the control group brings a significant change.

The pelvic floor muscles constitute the core muscles of the pelvis; the study was designed to investigate the effect of pelvic floor exercises on symptoms and bother-

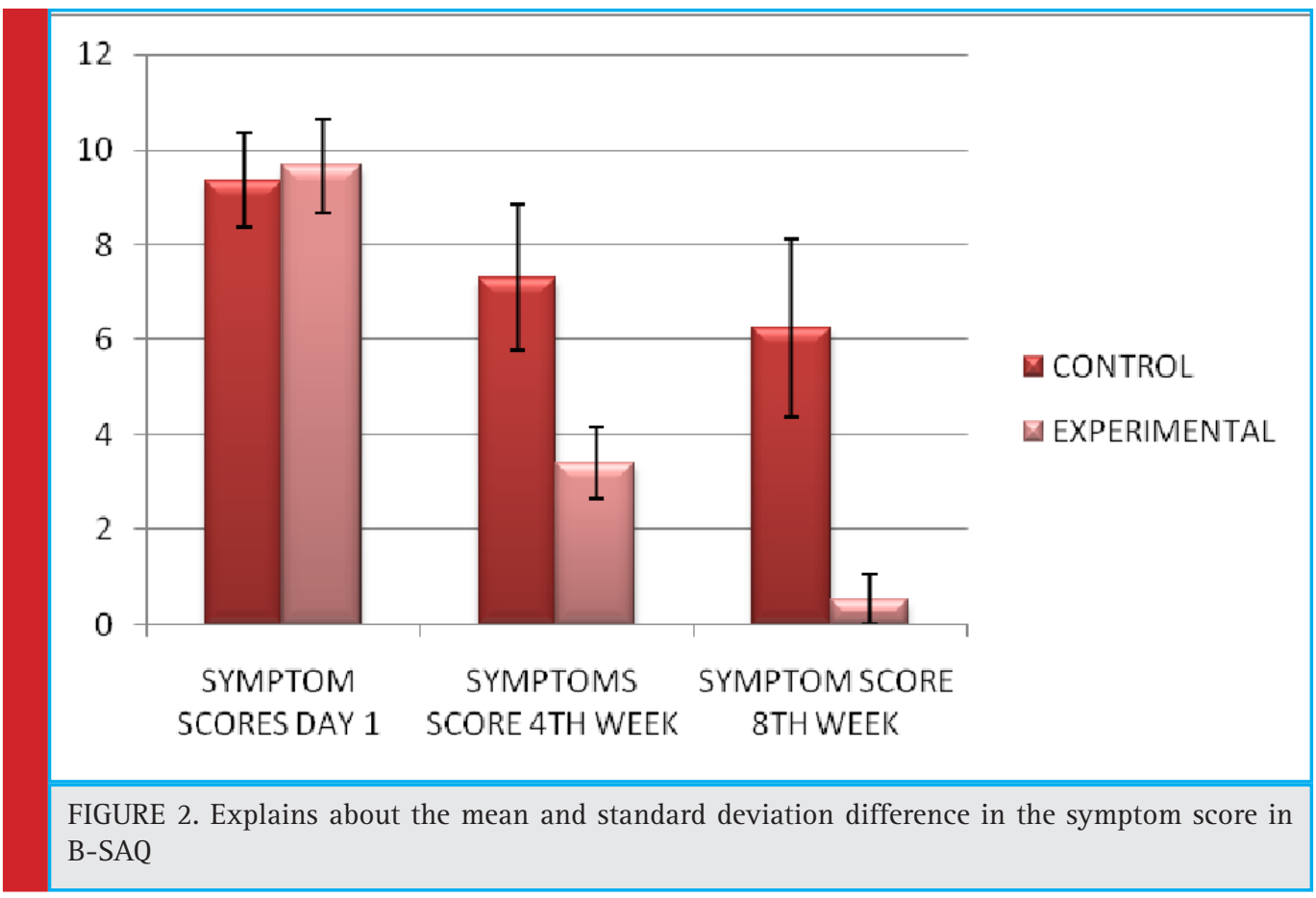




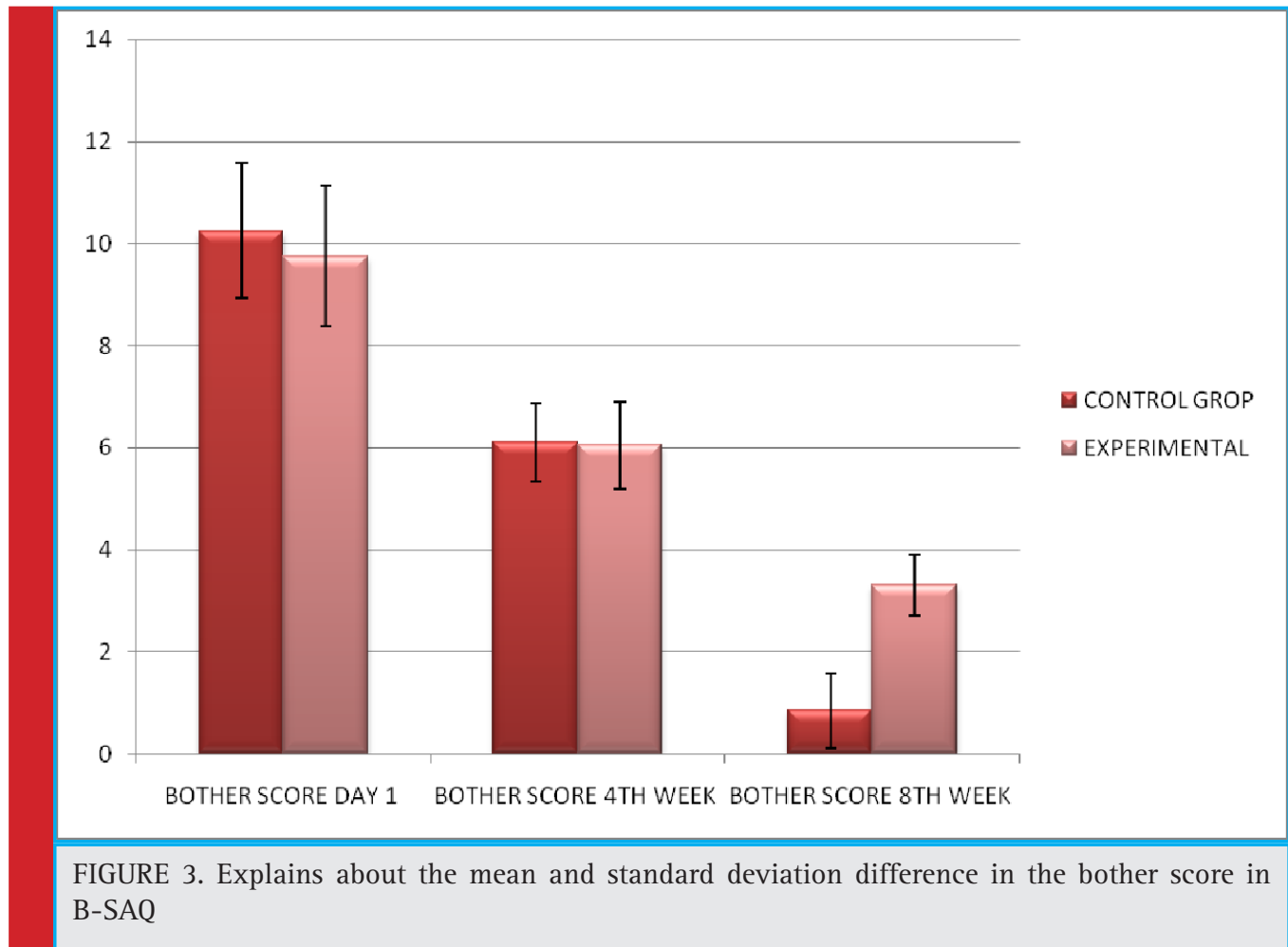

ness caused by urinary incontinence and achieving continence level were marked through pad test. There was a significant improvement in the symptoms scores of experimental group in contrast with the control group. The bother score when compared in both the groups at baseline and $4^{\text {th }}$ week there was no statistical significant difference seen. The present study showed a significant decrease in symptoms and levels of incontinence, also there was an improvement reduction in frequency, urgency and nocturia in experimental group when compared to the control group in a 12 weeks protocol. Supervised protocol for pelvic floor exercises proved to be a most effective method for improvement in the symptoms in urinary incontinence. In a study by Sharon et al. (2010), it has been explained that this severity impacted the quality of life it was found that 30 $\%$ of female subjects and 18\% of male subjects were reported with micturition dribbling and severity from mild to moderate, also female were more affected than men associated with other variable representing increase in the micturition dribble .this study showed that the increase in symptoms were disturbing quality of life. A significant improvement was seen in quality of life and self esteem frequency of urination and amount of urine, (Johromi et al. 2014).

In a study performed by Ali et al. (2011) the effect of Kegels exercises on women aged between 25-54 years suffering from urinary incontinence was carried out, it was found that the average score before administering the exercises was 53.15 and after the treatment there was a significant improvement to 73.82, bringing the significant level to $p=0.0001$. A study performed by another researcher on 30 patients affected with urinary incontinence in Imam Reza and Gharazi hospital at Sirjan city Iran with a aim to investigate the effect of kegels exercises on incontinent female subject for 3 month showed

\begin{tabular}{|c|c|c|c|c|c|}
\hline Source of Variation & $\mathrm{DF}$ & SS & MS & $\mathrm{F}$ & $\mathrm{P}$ \\
\hline Between Subjects & 19 & 59.500 & 3.132 & & \\
\hline Between Treatments & 5 & 1254.367 & 250.873 & 230.716 & $<0.001$ \\
\hline Residual & 95 & 103.300 & 1.087 & & \\
\hline Total & 119 & 1417.167 & 11.909 & & \\
\hline
\end{tabular}


a significant improvement in reduction frequency of urination and was lessened by $30 \%$ concluded that kegels exercises can become a suitable method to treat urinary incontinence, (Baba et al. 2006). Through the present study we explain that it is better to do pelvic floor exercises under supervision of a physiotherapist, learning of the correct technique of exercises to strengthen the muscle for required results, 2 months of exercises protocol results in the improving the strength and symptoms.

Limitation of the study : Considering small sample size difficult to draw and firm conclusion Explaining and recognizing the correct pelvic floor muscle and skilled exercises learned by patient as treatment can result in positive effect but if not can result in negative effect increasing frequency of urination and dribbling.

\section{CONCLUSION}

Pelvic floor muscle exercises can be considered as an empowerment methods for improvement in the symptoms and bother score also improving on pelvic floor muscle strength. So it is recommended that these types of exercising protocol can be utilized in improving the strength and quality of life in patient suffering from incontinence

\section{REFERENCES}

Aarthi M, Sankarganesh A. and Sivakumar V.P.R. (2018) Interferential Therapy Versus Pelvic Floor Exercise for the Management of Stress Urinary Incontinence in Women. Journal of physiotherapy research Vol.2 No.1(1): Pages 1-7

Baba Mohammadi H and Khalili H. (2006) The effect of pelvic floor exercise in the treatment of urinary incontinence in the elderly, Ghrzy and Imam Reza hospitals in Sirjan city. Yazd Journal of Medical Sciences and Health Services. Vol 3: Pages 61.

Bhanupriya, Singh N. and Goel N.(2015) Prevalence and Risk Factors of Urinary Incontinence Among Women Delivering in a Tertiary Care Center of Northern India. Obstetrics \& Gynecology International Journal.Vol.3 No. 4: Pages 1-4

Cavkaytar S.1., Kokanali M.K., Topcu H.O., Aksakal O.S. and Doğanay M. (2015) Effect of home-based Kegel exercises on quality of life in women with stress and mixed urinary incontinence. Vol.35 No.4:Pages 407-410

Dumoulin C., Hunter K.F., Moore K., Bradley C.S., Burgio K.L., Hagen S., Imamura M., Thakar R., Williams K. and
Chambers T. (2016) Conservative management for female urinary incontinence and pelvic organ prolapse review 2013: Summary of the 5th International Consultation on Incontinence. Neurourology and urodynamics .Vol. 35 No.1:Pages 15-20

Ferreira P.H., Ferreira M.L. and Hodges P.W. (2004) Changes in recruitment of the abdominal muscles in people with low back pain ultrasound measurement of muscle activity. Spine. Vol. 29:Pages 2560-2566

Jahromi M.K., Ramezanli S., Taheri L. and Rahmanian A. (2014) Management of Stress Urinary Incontinence in Females with Diabetic Mellitus (Type 2). Journal of Diabetes \&t Metabolism. Vol 5 No.3: Pages 1-5

Kılic M. (2016) Incidence and risk factors of urinary incontinence in women visiting Family Health Centers; Springer Plus. Vol.5 No.1,1331:Pages 1-9

Luginbuehl H., Lehmann C., Baeyens J.P.,Kuhn A. and Radlinger L (2015) Involuntary reflexive pelvic floor muscle training in addition to standard training versus standard training alone for women with stress urinary incontinence: study protocol for a randomized controlled trial : BioMed Central Vol 16 No. 524: Pages 1-8

Malhotra N. and Chahal A. (2018) Effect of pelvic floor exercise on non-specific lower back pain in post-partum women Biosciences Biotechnology Research Communications Vol.11 No. 3: Pages 469-475

Sahai A, Dowson C., Cortes E., Seth J., Watkins J., Khan M.S., Dasgupta P., Cardozo L., Chapple C., De- Ridder D., Wagg A. and Kelleher C. (2014) Validation of the bladder control selfassessment questionnaire (B-SAQ) in men. British Journal of Urology Vol.113 No.5: Pages 783-788

Sapsford R.R, Hodges PW, Richardson CA, Cooper DH, Markwell SJ and Jull GA. Co-activation of the abdominal and pelvic floor muscles during voluntary exercises. Neurourology and Urodynamics. Vol. 20: Pages 31-42.

Shah Ali S. H, Kashanian M. and Azari A. (2011) The effect of pelvic floor exercises on quality of life in women with stress urinary incontinence. The Bring knowledge Magazine. Vol.15: Pages 159-162

Sinclair A.J. and Ramsay I.N. (2011) The psychosocial impact of urinary incontinence in women. The Obstetrician \& Gynaecologist.Vol. 13: Pages 143-148.

Tennstedt S.L, Chiu G.R., Link C.L., Litman H.J., Kusek J.W. and McKinlay J.B.(2010) The effects of severity of urine leakage on quality of life in Hispanic, white and black men and women: the Boston community health (bach) survey. Urology. Vol. 75No1: Pages 27-33 